\title{
MONTE-CARLO SIMULATIONS
}

\author{
MIREK GIERSZ \\ Nicolaus Copernicus Astronomical Centre, \\ Polish Academy of Sciences, \\ Bartycka 18, 00-716 Warsaw, \\ Poland
}

\begin{abstract}
The revision of the Stodólkiewicz's Monte-Carlo code is presented. It treats each superstar as a single star and follows the evolution and motion of all individual stellar objects. The first calculations, for equalmass $N$-body systems with three-body energy generation accordingly to Spitzer's formulae, show good agreement with the direct $N$-body calculations for $N=2000$ and 10000 particles.
\end{abstract}

\section{INTRODUCTION.}

Our knowledge about the stellar content, kinematics and the influence of the environment on observational features of globular clusters and even richer stellar systems are increasing dramatically (as we could learn, for example, from talks presented on this conference). First, observations are reaching the point where segregation of mass within globular clusters can be observed directly and quantitatively. Second, observations have revealed that clusters with dense (collapsed) cores are relatively more concentrated to the galactic center than uncollapsed ones. Thus the influences of the environment and mass spectrum are crucial for cluster evolution. Third, recent observations show that many different and fascinating types of binaries and binary remnants are present in abundance in globular clusters. Binaries, in addition to being a diagnostic of the evolutionary status of clusters, are directly involved in the physical processes of energy generation, providing the energy source necessary to stop the core collapse and then drive the core expansion. So, to model the evolution of real stellar systems and make meaningful comparison with observation one has to take into account the 
complex interactions between stellar evolution and stellar dynamics. Of course all these demands can be easily fulfilled by direct $N$-body models. But they are very time-consuming and they need a special-purpose hardware to be run efficiently (Makino 1995, Taiji 1995). Another possibility is to use a code, which is very fast and can properly reproduce the standard relaxation process, and at the same time provides a clear and unambiguous way of introducing all the physical processes, which are important during globular cluster evolution. Monte-Carlo codes, which use a statistical method of solving the Fokker-Planck equation, provide all the necessary flexibility. They were developed by Spitzer (1975, and references therein) and Hénon (1975, and references therein) in the early seventies, and substantially improved by Marchant \& Shapiro (1980, and references therein) and Stodółkiewicz (1986, and references therein). Unfortunately, lack of fast computers at that time and development of the direct Fokker-Planck and gaseous models contributed to the abandonment of this method. But recent developments in computer hardware now make it possible to run a Monte-Carlo code efficiently. The great advantages of this method, besides of its simplicity and speed, are connected with the inclusion of anisotropy, and with the fact that added realism does not slow it down.

The Monte-Carlo code can have another possible use. Despite the simplified nature of continuum models (Fokker-Planck and gaseous models) they will continue for a while to be the most commonly used codes for stellar dynamical evolution. The Monte-Carlo models can be used to optimize free parameters of continuum models and to check their validity as it was done in the comparison between small $N$-body simulations and continuum ones (Giersz \& Heggie 1994ab, Giersz \& Spurzem 1994). This procedure should further increase our confidence of the results obtained in Fokker-Planck or gaseous simulations.

\section{MONTE-CARLO METHOD.}

\subsection{BASIC IDEAS.}

The Monte-Carlo method can be regarded as a statistical way of solving the Fokker-Planck equation. The basic idea behind the Monte-Carlo method is as follows. During the time interval $\Delta t$, much smaller than the relaxation time, the fluctuating gravitational field (connected with distant two-body interactions between stars) can be neglected in a first approximation and the system can be regarded as being in a steady state. However, the fluctuating gravitational field causes slow and random changes of the particle orbit parameters. This effect is small over $\Delta t$ but it builds up and becomes significant over the relaxation time scale and it has to be taken into account. To compute it, the standard Monte-Carlo tricks can be applied. The 
perturbation of a test star orbit is a random quantity, so only its statistical properties matter. The exact value of each perturbation is unimportant. The procedure to calculate perturbations is as follows: (1) instead of integrating the perturbations along the orbit, the perturbation is computed at a randomly selected point, (2) instead of considering the effect of all stars in the system, the perturbation is computed from a randomly chosen star, (3) the computed perturbation is multiplied by an appropriate factor in order to account for all the time points and all the system stars which have not been considered. If the procedure is correctly set up the evolution of the artificial system will be statistically the same as the evolution of the real one. For technical reasons (available computer memory and speed of computations) in all Monte-Carlo methods the whole system was divided into a certain number of superstars each consisting of a certain number of stars with the same mass, distance from the cluster center, radial and tangential velocities.

The way of implementing this basic strategy divides Monte-Carlo codes into three different groups; referred to as "Princeton", "Hénon" and "Cornell" methods (Spitzer 1987, and references therein). Each of these implementation was in the past successfully used in simulations of evolution of globular clusters and galactic nuclei.

\subsection{NEW IMPLEMENTATION OF THE STODÓŁKIEWICZ'S MONTE-CARLO SCHEME.}

The real power of Monte-Carlo codes was demonstrated by Stodółkiewicz $(1982,1985,1986)$. He substantially improved Hénon's version of MonteCarlo code by adding individual time-step scheme and a special procedure, which makes the total energy conservation much more strict. He used the code to model the evolution of globular clusters influenced by the following processes: formation of binaries by dynamical and tidal interactions, interactions between binaries and field stars and between binaries themselves, collisions between stars, stellar evolution, the tidal field of the Galaxy and tidal shocks. These were unique calculations, and have never been repeated or superseded by anybody. Excellent and very detailed description of the Stodółkiewicz's code can be found in Stodólkiewicz $(1982,1986)$.

Unfortunately the Stodólkiewicz's method is not suitable to correctly represent the very center of the system. In the core, as a result of the collapse, the density in a small and ununiform region reaches high values. This area is represented by only a few superstars. Therefore the statistical properties of this region are very poorly described. Moreover, superstars which constitute the core take part in processes which are responsible for energy generation and creation, in direct star interactions, many different 
and fascinating types of binaries, binary remnants and coalesced stars. In the new code, in order to properly describe this region and these processes, each superstar is treated as a single star and evolution and motion of all individual objects are followed. This improvement is possible only due to enormous increase of speed and memory in present day general-purpose computers.

Note, that individual treatment of all objects in the system enables, for example, to investigate influence of primordial binaries on the system evolution. In the Stodółkiewicz's method all binaries or coalesced stars take part only in relaxation process. They are neglected in computation of gravitational potential, so the process of mass segregation is not properly described.

Basically, the improvement mentioned above is the only major change to the Stodółkiewicz's original code. Other changes are rather cosmetic and do not have any influence on code flow or implementation of physical processes.

\section{FIRST RESULTS}

The Monte-Carlo method contains several free parameters, which have to be adjusted in order to get the proper description of the system evolution. The best way of adjusting them is to compare the results of Monte-Carlo and direct $N$-body simulations. The same strategy was used to optimize free parameters of the continuum models (Giersz \& Heggie 1994ab, Giersz \& Spurzem 1995).

The good statistical quality data for single mass $N$-body simulations are available only for $N=250,500,1000,2000$ and 10000 (Giersz \& Heggie 1994ab, Giersz \& Spurzem 1994, Aarseth \& Spurzem 1995). Simulations with $N=2000$ are the best for our purposes. They cover the system evolution up to twelve collapse times and consist of sixteen separate runs.

Most of the Monte-Carlo simulations were run for systems consisting of 2000 equal mass particles, but additionally a few simulations were performed with $N=10000$ and 30000 . Pilot runs have shown that the best choice of free parameters is practically the same as chosen by Stodółkiewicz (1982). The results discussed bellow $(N=2000)$ were averaged over 25 simulations, each having the same initial parameters but with different sequence of random numbers used to initialize the positions and velocities of the stars.

During the phase of core collapse the $N$-body and Monte-Carlo models follow each other very closely (Fig. 1). The first differences start to build up around the time of the core bounce. This is, particularly well, visible for the middle and outer Lagrangian radii. In the Monte-Carlo simulations the 


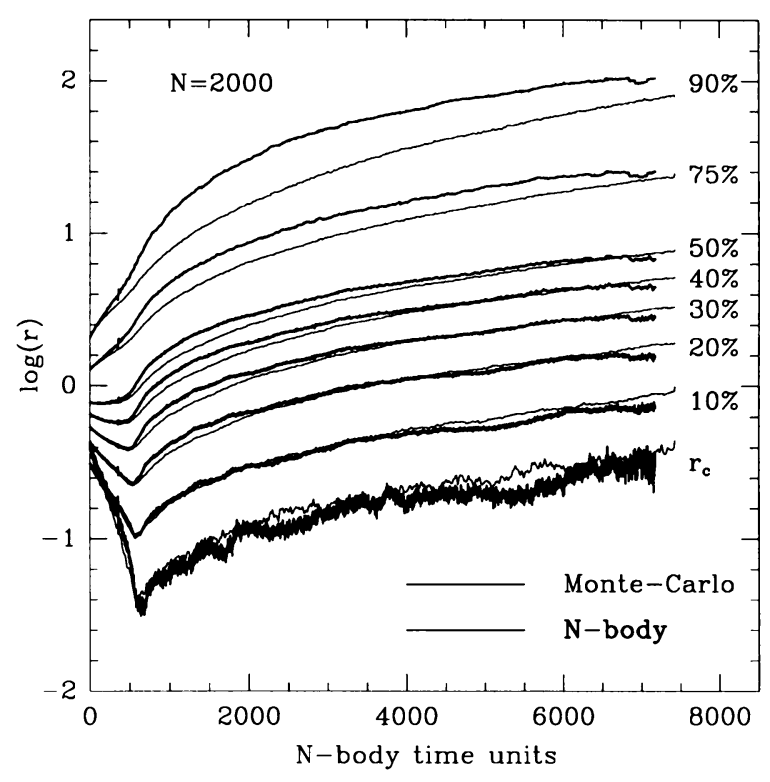

Figure 1. Evolution of Lagrangian radii for $N=2000$ for Monte-Carlo model (solid line) and $N$-body model (thick solid line). The core radii for both model are also shown.

rate of system expansion is slower just after the core bounce and slightly faster for the advanced post-collapse evolution. For the inner parts of the system, up to the Lagrangian radius containing $10 \%$ of the total mass, the evolution for both models is very similar. Only the core collapse is slightly deeper in the $N$-body model. It is worth to note that the collapse time for both models is practically the same. This further confirms the value of $\gamma=0.11$, in the Coulomb logarithm, obtained by comparison of $N$-body and continuum models (Giersz \& Heggie 1994a).

Similarly as for the inner Lagrangian radii the anisotropy for the inner half of the system is very well reproduced by the Monte-Carlo model (Fig. 2 ). For the outermost part of the system (Lagrangian radii containing more than $90 \%$ of the total mass) the anisotropy in the $N$-body simulations is larger from the very beginning of the core expansion. This suggests that in the $N$-body simulations halo is developed faster than in the Monte-Carlo ones. At least part of the differences in the anisotropy labeled by $75 \%$ can be explained by the way of anisotropy computation. For the $N$-body simulations the displayed anisotropy is computed for shell between $50 \%$ and $75 \%$ Lagrangian radii, while for the Monte-Carlo simulations for shell between $70 \%$ and $75 \%$ Lagrangian radii. Because the anisotropy increases with radius, so it should be slightly larger for the Monte-Carlo model than for the $N$-body one. 


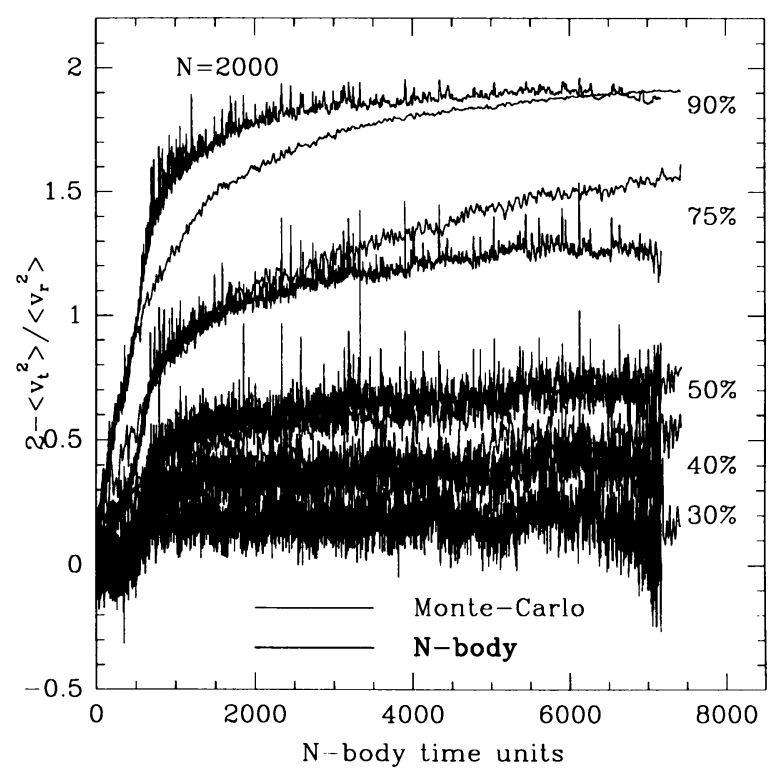

Figure 2. Evolution of the anisotropy for $N=2000$ for Monte-Carlo model (solid line) and $N$-body model (thick solid line).

The clue to explain the differences between the $N$-body and MonteCarlo models gives Fig. 3, which shows the energy balance. There are three main differences between models visible in this figure. Firstly, the energy carried out by single star escapers, $E_{\text {ext }}^{e s}$, is much larger in the Monte-Carlo simulations. This is connected with the fact that the number of escapers is larger by about $30 \%$ and that the escapers connected with interactions between three-body binaries and field stars are more numerous in the MonteCarlo model. The larger number of escapers in the Monte-Carlo simulations is mainly connected with the fact that stars are immediately removed from the system, while in the direct $N$-body simulations they are only removed when they are further than ten times the half-mass radius. Secondly, binaries start to form slightly earlier and the number and the total internal energy of escaping binaries, $E_{i n t}^{e b}$, are larger in the Monte-Carlo simulations. Thirdly, around the time of core bounce, the total internal binding energy of the three body binaries bound to the system, $E_{\text {int }}^{b}$ is smaller in the MonteCarlo simulations. This is despite that the number of binaries bound to the system is practically the same in both models. Too early energy generation by three-body binaries makes the core collapse less deep and as well less abrupt expansion of the outer parts of the system. On the other hand in the $N$-body simulations binaries, which stay in the core, harden to higher binding energies than in the Monte-Carlo simulations. So they can produce 


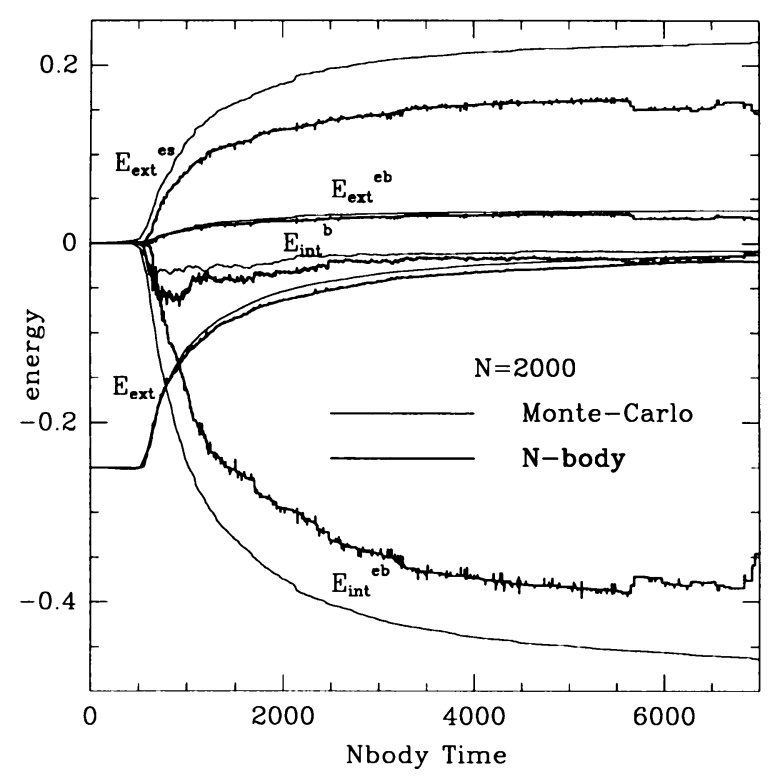

Figure 3. Energy balance for $N=2000$ for Monte-Carlo model (solid line) and $N$-body model (thick solid line). The meaning of the symbols is given in the text.

more stars, which penetrate, on nearly radial orbits, the outermost parts of the system partially contributing to larger anisotropy. Smaller binding energy of the system, $E_{\text {ext }}$ for Monte-Carlo simulations is connected with the fact that there are substantially more escapers than in the $N$-body simulations.

Comparison between the Monte-Carlo simulations and the $N$-body ones for $N=10000$ shows, basically, the same features as in the case of $N=$ 2000 .

The gravothermal oscillations are the most pronounced feature of the post-collapse evolution of $N$-body systems with number of stars greater than a few thousands. They were observed in gas (Bettwieser \& Sugimoto 1984, Goodman 1987, Heggie \& Ramamani 1989), Fokker-Planck (Cohn et al 1986, Cohn, Hut \& Wise 1989, Gao et al 1991) and recently in $N$-body simulations (Makino 1995). The lowest value of $N$ for which the gravothermal oscillations begin to show up is uncertain. But recent pilot $N$-body simulation of system consisting of 16000 particles ( Makino 1995) shows clear oscillations. All this suggests that gravothermal oscillations should, as well, be present in the Monte-Carlo simulations, at least for $N=30000$.

In Fig. 4 the evolution of the logarithm of the central density for $N=$ 2000,10000 and 30000 , respectively is shown. It seems that gravothermal oscillations are visible in system with 30000 particles and there are some 


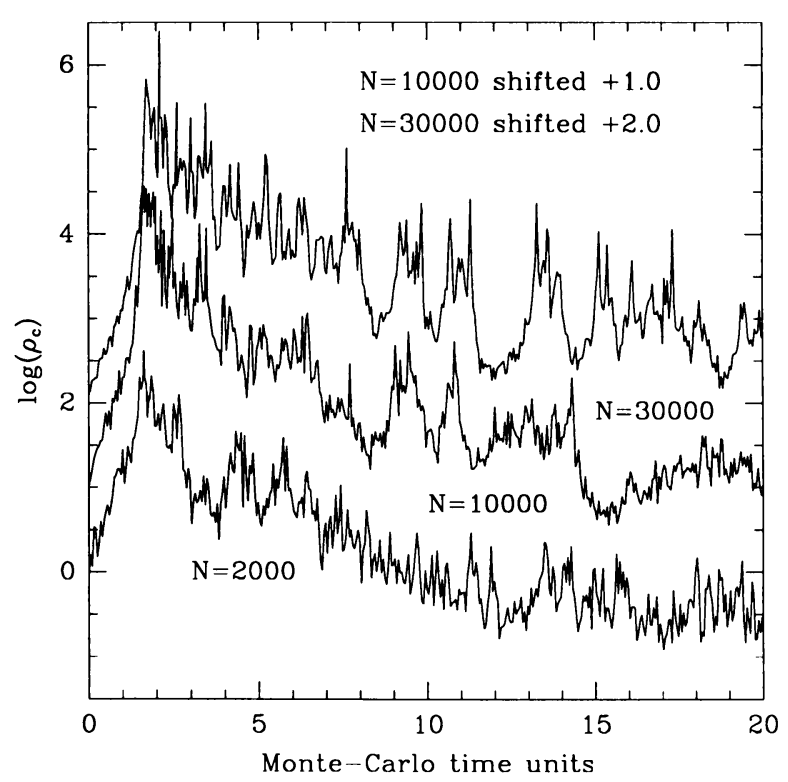

Figure 4. Evolution of the central density for $N=2000,10000$ and 30000. Data for $N=10000$ is shifted up by 1 in the logarithm of the central density. Data for $N=30000$ is shifted up by 2 in the logarithm of the central density .

signs of gravothermal oscillations for $N=10000$. This conclusion is somewhat ambiguous, because binary activities can give similar behaviour of the central density. But amplitudes of oscillations connected with binary activities are usually smaller than observed in 10000 and 30000 body simulations. Evidently more simulations with broader range of $N$ are needed to clarify this problem and to get a better agreement with the direct $N$-body simulations.

At the end I would like to show an unpublished (in international astronomical literature) result of the Stodółkiewicz Monte-Carlo simulations of two-component system with $10^{5}$ stars, performed in 1986 (Fig. 5) The evolution of central density for unevolved and evolved stars is shown together with the evolution of the total central density. The total central density (dominated by evolved stars) behaves in manner characteristic for gravothermal oscillations. I think that this is the remarkable result obtained well before the gravothermal oscillations were confirmed and widely accepted by the astronomers.

Finally, a few words about the efficiency of the new code. The calculation of $N=2000,10000$ and 30000 models takes about 2, 20 and 130 hours, respectively. The theory predicts a linear increase of computing time with $N$. This is connected with the fact that the most time consuming part of the code is the computation of the potential, which is proportional to $N$ 


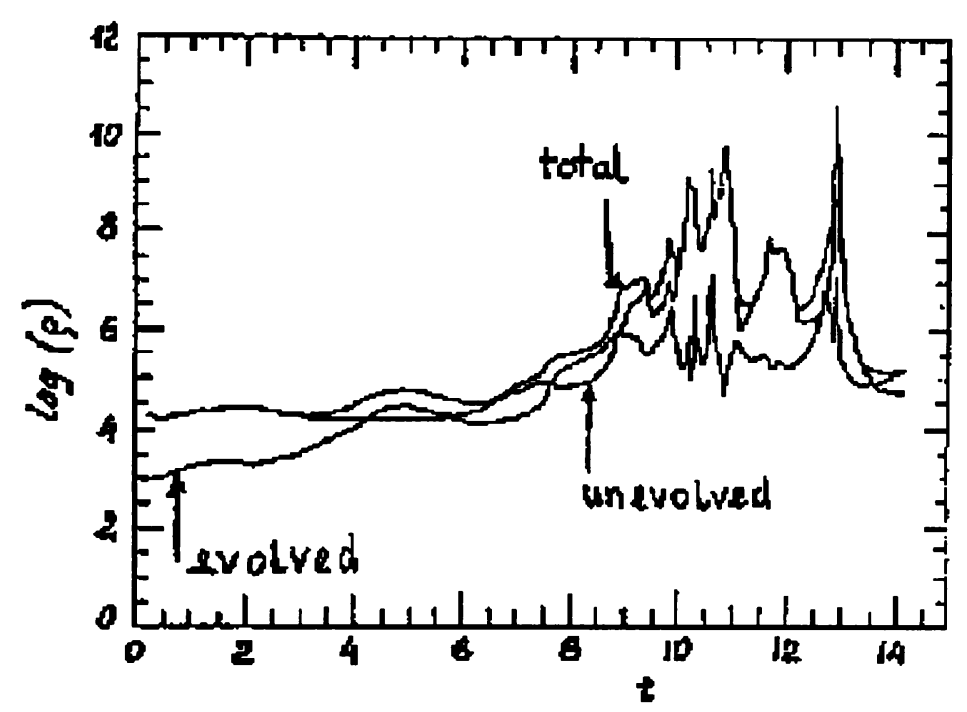

Figure 5. Evolution of the central density for Stodółkiewicz Monte-Carlo model for $N=10^{5}$ two component system. Arrows show the densities for evolved, unevolved stars and the total density.

due to spherical symmtery of the system. The real calculations give slightly steeper dependence on $N$. It seems that this is connected with the fact that larger systems have more density peaks, which implies smaller time steps in order to properly resolve the system evolution. The high speed of the code makes it possible to run several different models to improve statistical quality of the data and investigate influence of different physical processes on cluster evolution.

\section{FUTURE DEVELOPMENTS OF THE NEW MONTE-CARLO CODE}

The new Monte-Carlo code presented in the previous section can be treated only as a first approximations. Several important physical processes have to be included to make the simulations of stellar systems more realistic. The final code will contain the following physical processes:

- formation of binaries due to dynamical and tidal interactions,

- primordial binaries,

- stellar evolution,

- tidal field of Galaxy, and tidal shocks connected with crossing of the galactic plane and with large molecular clouds,

- collisions between stars, 
- interactions between binaries and stars and between binaries themselves.

In the first stage of the code development (nearly completed) all processes connected with interactions between objects will be modeled using analytical cross sections available in the literature. This will allow the code to be tested and make possible comparison with continuum models.

In the next stage interactions between groups of three and four (finite size) stars will be modeled by numerical integrations of their orbits (the first attempts are tested now). This more realistic approach ensures that processes of energy generation (the most important factor in the dynamical evolution of globular clusters) will be modeled more closely.

The final stage will be the inclusion of a detailed 3-D. hydrodynamical modeling of collisions between stars. This will be done by use of Smooth Particle Hydrodynamics (SPH) for a limited number of particles per star (a few hundred). This will allow for the first time close comparison between numerical models and observations of real globular clusters. I refer here to observations of various, peculiar objects like blue stragglers and milliseconds pulsars, which can be formed during collisions and encounters between stars.

\section{References}

Aarseth S.J. \& Spurzem R., 1995, in preparation

Bettwieser E. \& Sugimoto D., 1984, MNRAS, 208, 498

Cohn H., Wise, M.W., Yoon T.S., Statler T.S., Ostriker J., Hut P., 1986, in Hut P., McMillan S., eds., The Use of Supercomputers in Stellar Dynamics, New York: Springer, p. 206

Cohn H., Hut P., 1989, ApJ, 226, 1087

Gao B., Goodman J., Murphy B., Cohn H., 1991, ApJ, 370, 567

Giersz M. \& Heggie D.C., 1994a, MNRAS, 268, 257

Giersz M. \& Heggie D.C., 1994b, MNRAS, 270, 298

Giersz M. \& Spurzem R., 1994, MNRAS, 269, 241

Goodman J., 1987, ApJ, 313, 576

Hénon M., 1971, Astrophys. Sp. Sci., 14, 151

Hénon M., 1975, in Hayli A., ed, Dynamics of Stellar Systems, Reidel: Dordrecht, p.133

Heggie D.C., \& Ramamani N., 1989, MNRAS, 237, 757

Makino J., 1995, this volume

Marchant A.B, \& Shapiro S.L., 1980, ApJ, 239, 685

Spitzer L., Jr., 1975, in Hayli A., ed, Dynamics of Stellar Systems, Reidel: Dordrecht, p.3

Spitzer L., Jr., !987, Dynamical Evolution of Globular Clusters. Princeton Univ. Press, Princeton, p.77

Stodółkiewicz J.S., 1982, Acta Astr., 32, 63

Stodółkiewicz J.S., 1985, in Goodman J. \& Hut P., eds, Dynamics of Star Clusters, Reidel:Dordrecht, p.361

Stodółkiewicz J.S., 1986, Acta Astr., 36, 19

Taiji M., 1995, this volume 